\title{
Predicting Bulk Damage in NIF Triple Harmonic Generators
}

\author{
M. Runkel \\ W. Williams \\ J. DeYoreo
}

This paper was prepared for submittal to the 30th Boulder Damage Symporium: Annual Symposiun on

Optical Materials for High Power Lasers Boulder, Colorado

September 28 - October 1, 1998

\section{September 18, 1998}

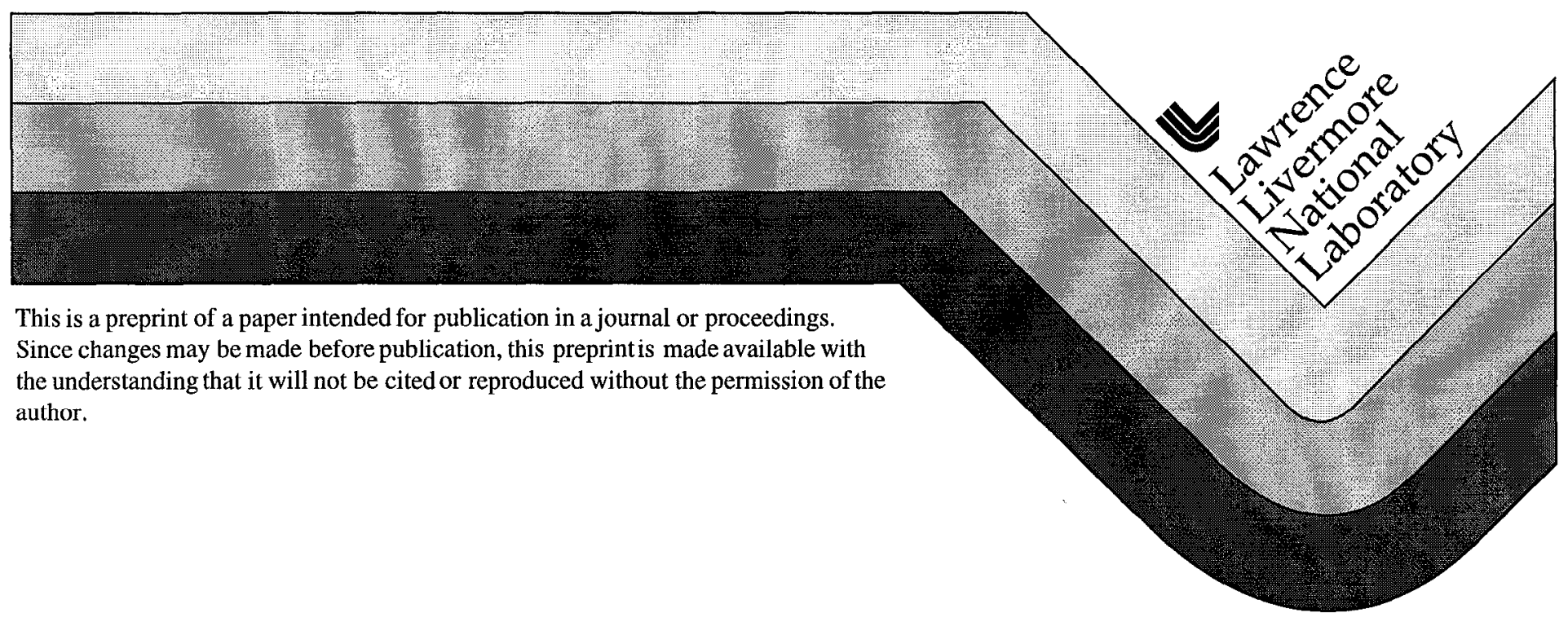




\section{DISCLATMER}

This document was prepared as an account of work sponsored by an agency of the United States Government. Neither the United States Government nor the University of California nor any of their employees, makes any warranty, express or implied, or assumes any legal liability or responsibility for the accuracy, completeness, or usefulness of any information, apparatus, product, or process disclosed, or represents that its use would not infringe privately owned rights. Reference herein to any specific commercial product, process, or service by trade name, trademark, manufacturer, or otherwise, does not necessarily constitute or imply its endorsement, recommendation, or favoring by the United States Government or the University of California. The views and opinions of authors expressed herein do not necessarily state or reflect those of the United States Goverrument or the University of California, and shall not be used for advertising or product endorsement purposes. 


\title{
Predicting bulk damage in NIF triple harmonic generators
}

\author{
M. Runkel, W. Williams, J. DeYorco \\ Lawrence Livermore National Laboratory \\ P.O. Box 808, L-250 \\ Livermore, CA 94550 \\ Phone: (925)-424-2210 \\ FAX: (925)-422-3361 \\ E-mail: runkel1@llnl.gov
}

\begin{abstract}
Recently reported experiments ${ }^{12}$ have investigated the statistics of laser damage in KDP and KD*P. Automated damage tests have allowed cumulative failure and damage probability distributions to be constructed. Large area tests have investigated the feasibility of on-line laser conditioning and damage evolution for tripler harmonic generation (THG) crystals on the National Ignition Facility (NIF). These tests have shown that there is a nonzero probability of damage at NIF redline fluence $\left(14.3 \mathrm{~J} / \mathrm{cm}^{2}, 351 \mathrm{~nm}, 3 \mathrm{~ns}\right)$ and that the damage pinpoint density evolves exponentially with fluence.
\end{abstract}

In this paper, the results of these tests are used in conjunction with model spatial profiles of the NIF beam to predict the level of damage created in the THG crystal. A probabilistic calculation based on the overlap of the beam fluence and damage probability distribution shows that the overall damage probability is less than $3 \%$ for well-conditioned, high quality KDP/KD*P crystals of conventional or rapid growth. The number density of generated pinpoints has been calculated by mapping the damage evolution curves onto the NIF model profile. This shows that the number of damage pinpoints generated in high fluence portions of the NIF beam will be low for well-conditioned THG crystals. In contrast, unconditioned triplers of the same material will exhibit an increase in pinpoint density of greater than $20 \mathrm{X}$.

To test the validity of these calculations a $37 \mathrm{~cm}$, conventionally grown KD*P tripler from the Beamlet laser was scatter mapped for bulk damage. The tripler had been exposed to NIF-like fluences during its operational lifetime on Beamlet and exhibited very low levels of bulk pinpoint damage, essentially supporting the predictions based on tests and modeling.

Keywords: KDP, KD*P, bulk damage, damage prediction

\section{INTRODUCTION}

The National Ignition Facility (NIF), currently under construction at Lawrence Livermore National Laboratory (LLNL) represents the next generation in Inertial Confinement Fusion lasers. Designed to achieve laser fusion, the projected 1.8 MJ, $351 \mathrm{~nm}$ output has placed severe damage performance requirements on the beamline optics. In particular, the KD*P triple harmonic generation crystals (THG) are considered the system fuse because their damage performance could not meet the design specifications unless the crystals were laser (pre)conditioned. Because of this, a vigorous research and development effort is currently underway to determine how to grow and process $\mathrm{KD}^{*} \mathrm{P}$ triplers to yield maximum laser damage performance in a $50 \times 50 \mathrm{~cm}^{2}$ crystal.

In conjunction with the KDP development effort, a broad spectrum of damage test methods and modeling tools have allowed the investigation of tripler damage performance in a NIF-like beam environment to be investigated. This question is central to development of functional damage threshold and lifetime specifications for NIF triplers. Automated and large area damage test facilities coupled with computer programs to calculate NIF spatial profiles are the core components of this work. In sections 2 and 3 of this paper, the role of automated damage test results and NIF model spatial profiles will be described. In section 4, the results of reliability analysis calculations for determining the probability of failure will be discussed. Appendix 1 gives a more detailed derivation of the failure probability integral for gaussian distributions. Section 5 is devoted to damage evolution calculations. Large area pinpoint evolution data are used to determine the overall severity of damage generated by the NIF tripler spatial 
fluence profile. Section 6 summarizes the work and discusses the implications of these results for the determination of a functional damage specification for NIF triplers.

\section{AUTOMATED DAMAGE TESTING AND LASER INDUCED DAMAGE DISTRIBUTIONS}

The need for automated damage testing on KDP became apparent approximately 18 months ago when repeated testing of a large piece of rapidly grown KDP yielded inconsistent results using LLNL's (old) standard S/1 and R/1 test methods. It was eventually determined that these test methods did not provide an adequate statistical sampling of the damage distribution of the sample. Because of this, automation software and hardware were installed in the Zeus damage test lab for dedicated testing of KDP. Details reganding explicit system modifications are given in [1]. In short, the test procedure was modified to test one hundred sites to failure with ramped fluence exposures and in-situ damage detection diagnostics. This method provides information on precisely when damage occurs and allows the characteristic statistical behavior of the sample to be quantified. The reduced test data is typically displayed in a cumulative failure distribution (CFD) curve as a function of fluence where the individual damage thresholds of each test site are plotted in ascending order. Taking the derivative/histogram of the CFD yields information on the number of mechanisms contributing to damage as shown in Figure 1.

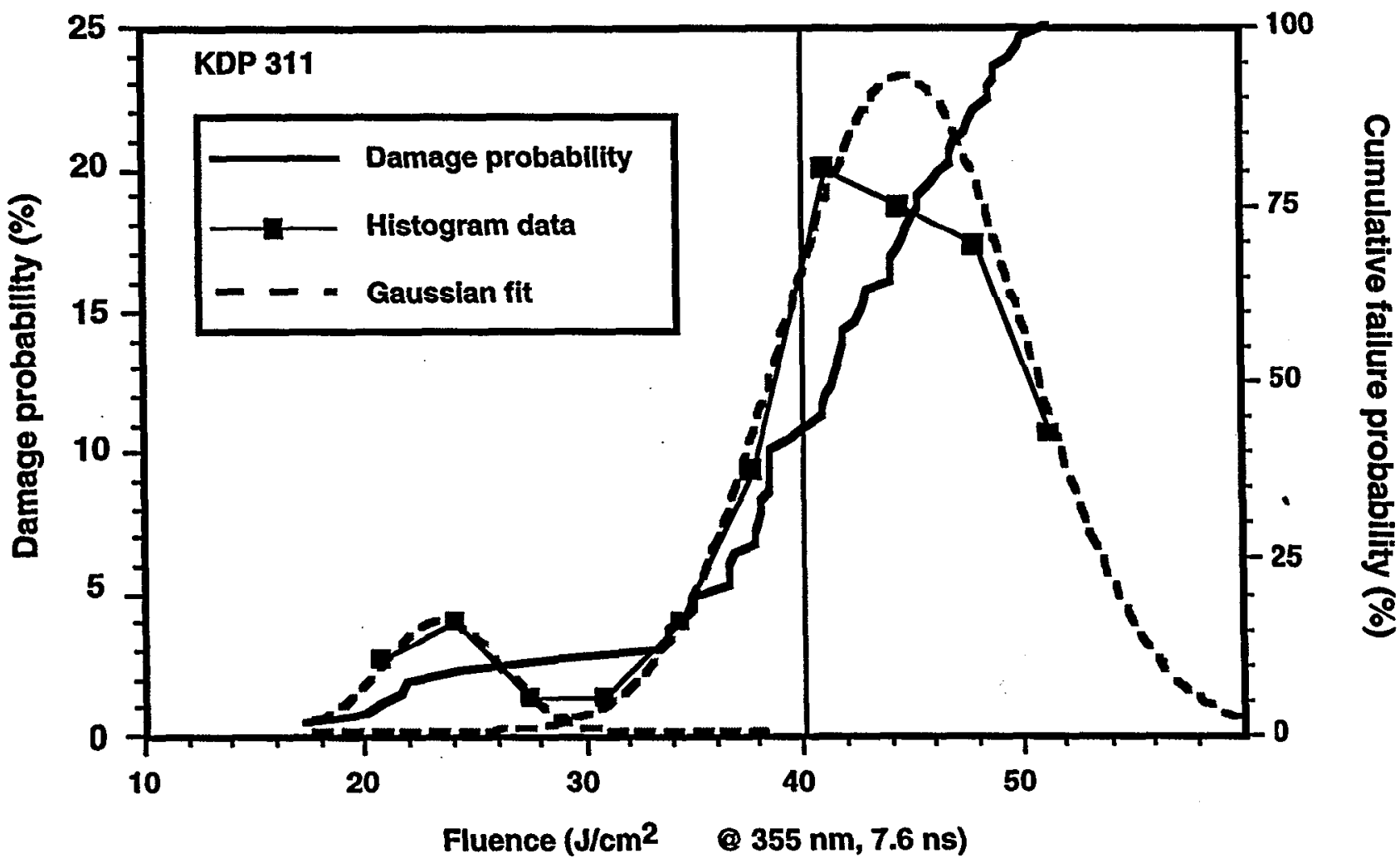

Figure 1. Cumulative failure probability curve and associated damage distribution for conventionally grown KDP sample 311. This material represents the highest threshold KDP ever tested at LLNL. Data shown for the $355 \mathrm{~nm}$, $7.6 \mathrm{~ns}$ pulsewidth of the Zeus automated damage test laser.

The figure shows numerous features associated with laser damage of $\mathrm{KDP}$ or $\mathrm{KD} * \mathrm{P}$. Histograms of the CDF typically can be fitted well using gaussian functions ${ }^{3}$. For high damage threshold material, there exists a tail on the CFD which indicates the existence of low fluence failure defects which have not been removed from the material. This class of defects is also exhibited by the smaller, low fluence peak in the histogram. As will be discussed in 
sections 4 and 5 , it is this low fluence peak which determines the damage behavior of the sample in the NIF operational fluence regime.

\section{NIF MODEL SPATIAL PROFILES}

The National Ignition Facility has been designed using a suite of computer programs which model beam energetics, growth of beam perturbations and laser beam propagations. A detailed discussion of these codes is beyond the scope of this paper, however, the details of the programs are well discussed in [4]. In order to calculate damage failure probabilities using reliability analysis techniques it is necessary to know the nature of the NIF beam statistics. The spatial fluence profile of the NIF beam at the output of the tripler crystal was modeled using the PROP925 computer program. This program is a full-featured optics propagation and laser simulation code written at LLNL. Included in the program are models for the majority of laser system components. Using these models the code can calculate the 2 dimensional complex electric field as it propagates through the laser system.

Figure 2 shows an intensity plot of the predicted spatial profile at the exit surface of the KD*P tripler for a full fluence NIF shot using the PROP92 program. The output consists of a $512 \times 512$ array covering $50 \times 50 \mathrm{~cm}^{2}$ so that the individual cells are $0.098 \times 0.098 \mathrm{~cm}^{2}$. The energy content of the beam in this simulation was $10.7 \mathrm{KJ}$ giving a 192 beam total of $2.05 \mathrm{MJ}$. The peak fluence in the beam is $12.85 \mathrm{~J} / \mathrm{cm}^{2}$. The average fluence of the distribution is $8.15 \pm 1.33 \mathrm{~J} / \mathrm{cm}^{2}$.
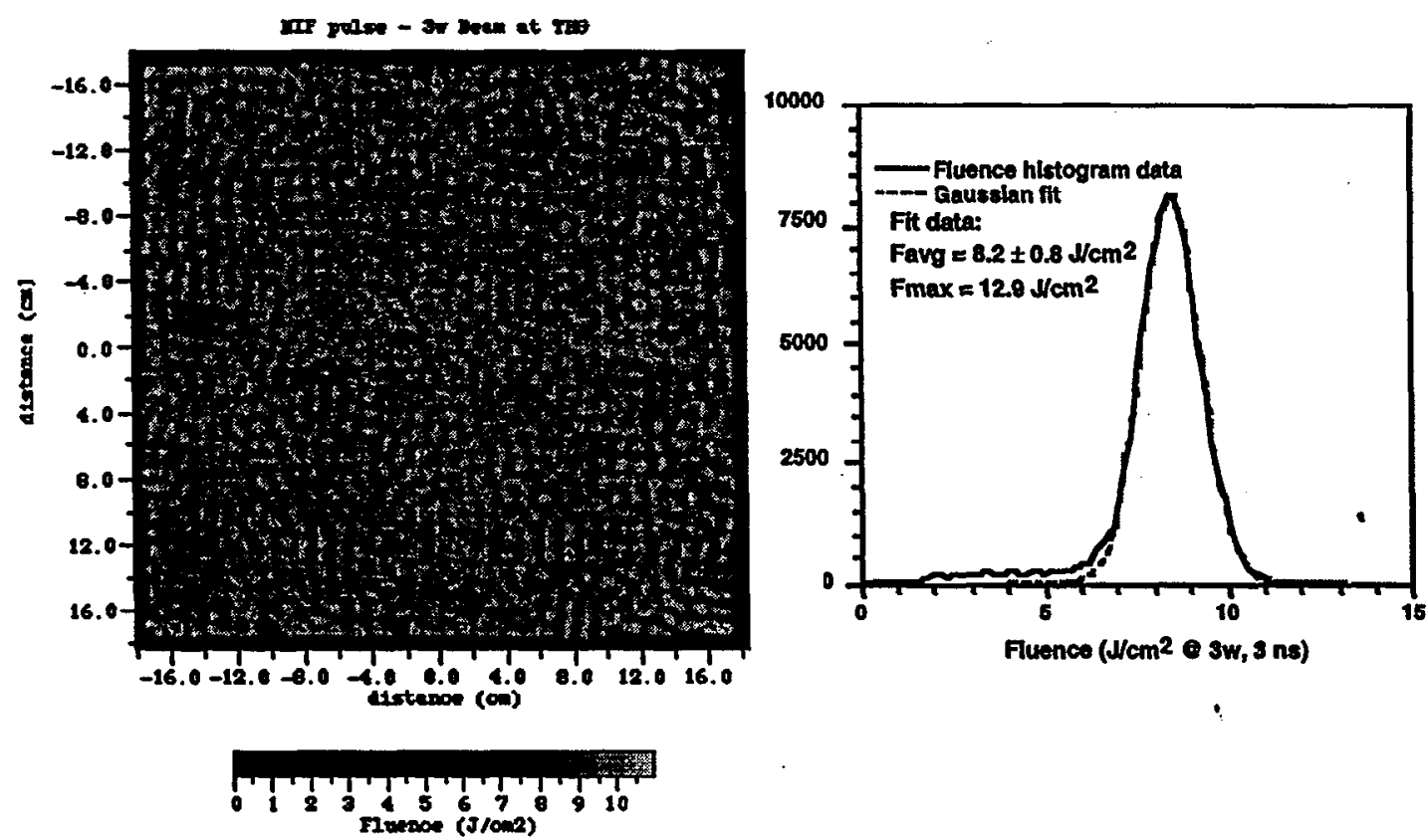

Figure 2. Spatial profile model of NIF beam fluence at output of the $\mathrm{KD} * \mathrm{P}$ tripler and the associated fluence distribution (histogram). The peak is fitted very well by a gaussian distribution with average of $8.2 \pm 0.8 \mathrm{~J} / \mathrm{cm}^{2}$.

Histogram analysis of the spatial profile yields the desired beam fluence distribution for the reliability analysis calculations to follow. Ignoring the low fluence tail in the distribution allows the peak to be fitted with a gaussian function with an average of $8.2 \pm 0.8 \mathrm{~J} / \mathrm{cm}^{2}$.

It should be noted that the data above are for the standard full fluence NIF shot and the $12.9 \mathrm{~J} / \mathrm{cm}^{2}$ peak fluence in the beam is $11 \%$ lower than the $14.3 \mathrm{~J} / \mathrm{cm}^{2}$ "redline" fluence for triplers. To examine the damage behavior of the triplers when exposed to a NIF shot with peak fluence at the redline, $11 \%$ was added to each fluence value in the spatial distribution. This resulted in a distribution of $9.1 \pm 1.5 \mathrm{~J} / \mathrm{cm}^{2}$ with a maximum fluence of $14.3 \mathrm{~J} / \mathrm{cm}^{2}$. The energy 
content in the beam then became $11.9 \mathrm{KJ}$ for a 192 beam total of $2.3 \mathrm{MJ}$. Fitting the peak of this distribution yielded a mean value (for the fit) of $9.4 \mathrm{~J} / \mathrm{cm}^{2}$ while the standard deviation remains unchanged at $0.8 \mathrm{~J} / \mathrm{cm}^{2}$.

\section{RELIABILITY ANALYSIS}

With knowledge of the beam fluence and damage statistics it is possible to make predictions regarding the reliability, or survivability, of a tripler when exposed to the model NIF pulse. The basic problem for determining the survivability of NIF optics is essentially one of determining the probability that local beam fluence is greater than the local damage threshold of the optic. This gives a measure of the damage/no damage response of the optic but does not provide any information regarding the nature or severity of damage once it does occur. A separate analysis (described in section 5) is required for this. Calculational tools from the field of reliability analysis ${ }^{6-8}$ are readily applied to the problem of determining the damage behavior of an optic when exposed to a large fluence distribution. For the problem at hand (Figure 3), it is clearly the overlap between the fluence and damage distributions which controls the survivability of the optic. For distributions which do not significantly overlap there is little chance that damage will occur. As the degree of overlap increases the probability for failure also increases.

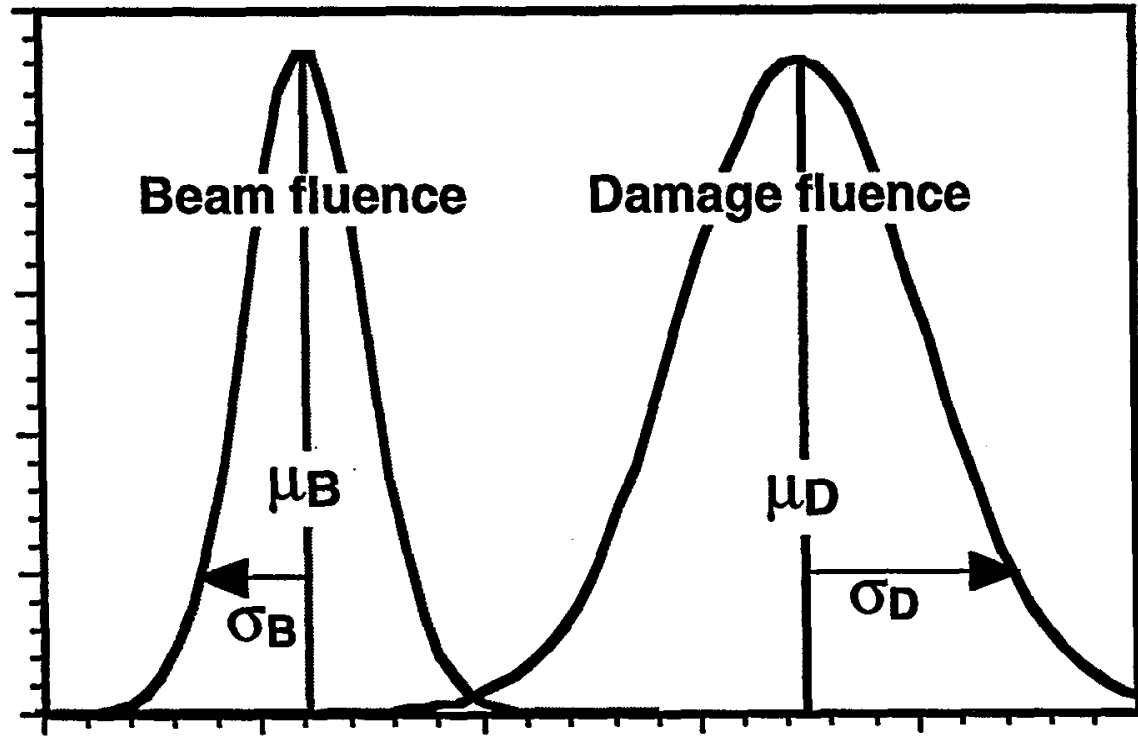

Fluence (Arb. units)

Figure 3. Plot showing the overlap between gaussian beam fluence and damage threshold distributions. The overlap between the distributions determines the overall probability of damage occurring on the optic.

\subsection{Damage failure probability integral for gaussian probability density functions}

Once best fit gaussian parameters have been obtained for the damage and beam fluence distributions the damage failure probability may be calculated. It is given by the expression

$$
P(B>D)=\frac{1}{\sqrt{2 \pi}} \int_{-\frac{\mu_{z}}{\sigma_{z}}}^{\infty} e^{-t^{2} / 2} d t
$$

for gaussian functions of the form 


$$
f(F)=f_{0} e^{-(F-\mu) / 2 \sigma^{2}}
$$

Here, $\mu$ is the mean of the distribution and $\sigma$ is the standard deviation, which gives a measure of the width of the distribution. The variable transformations $\mu_{\mathrm{z}}=\mu_{\mathrm{B}}-\mu_{\mathrm{D}}$ and $\sigma_{\mathrm{z}}{ }^{2}=\sigma_{\mathrm{B}}{ }^{2}+\sigma_{\mathrm{D}}{ }^{2}$ and

$$
t^{2}=\frac{\left(z+\mu_{D}-\mu_{B}\right)^{2}}{\left(\sigma_{B}{ }^{2}+\sigma_{D}{ }^{2}\right)}
$$

allow the integral to be rewritten in a form from which it can be evaluated from standard tables, or calculated numerically9. The subscripts $B$ and $D$ refer to the beam fluence and damage distributions respectively. Note that normalization of the gaussian functions is not required as the expression only depends on distribution means and standard deviations. A detailed derivation of (1) is given in Appendix 1.

With this result, data from Zeus damage distributions and NIF model profiles can be evaluated. The results are given below in Table 1 for the standard NIF and redline NIF pulses and rapid growth KDP sample 214 and conventional growth KDP 311. The damage fit parameters for the KDP samples are from fitting the low fluence defect distributions found in the tail of the CFD as shown in Figure 1. The values given for the NIF beam statistics are obtained from fitting the peak in the fluence distribution and do not account for the long low fluence tail (Figure 2).

Table 1. Results of failure probability calculations for NIF model beams and best rapid and conventional growth

\begin{tabular}{|c|c|c|c|c|}
\hline Sample & $\begin{array}{c}\text { Damage fit } \\
\text { parameters } \\
\left(\mu_{n} \pm \sigma_{0}\right) \\
\end{array}$ & $\begin{array}{l}\text { NIF beam statistics } \\
\qquad\left(\mu_{B} \pm \sigma_{B}\right)\end{array}$ & $\mathbf{t}$ & $\begin{array}{c}\text { Failure } \\
\text { probability } P_{t} \\
(\%) \\
\end{array}$ \\
\hline \multirow{2}{*}{$\begin{array}{c}214 \\
(L L 6-K D * P)\end{array}$} & \multirow{2}{*}{$11.0 \pm 1.0$} & $8.5 \pm 0.8$ (std. NIF shot) & 1.95 & 2.56 \\
\hline & & $9.4 \pm 0.8$ (redline shot) & 1.28 & 11.12 \\
\hline \multirow[t]{2}{*}{311} & \multirow[t]{2}{*}{$14.7 \pm 1.7$} & $8.5 \pm 0.8$ (std. NIF shot) & 3.30 & 0.05 \\
\hline & & $9.4 \pm 0.8$ (redline shot) & 2.80 & 0.26 \\
\hline
\end{tabular}
KDP (samples 214 and 311).

With the exception of firing a redline shot through a rapidly grown tripler, the results show the low probability of failure expected from exposing the best available rapid and conventional growth KDP to a full fluence NIF shot.

The data shows that sample 311 provides superior damage resistance, especially in the case of the hypothetical NIF redline shot. However, as will be shown in section 5 on damage evolution, the level of damage generated in high threshold, rapid growth material will be very low for the standard NIF shot.

\section{DAMAge PERformance BASEd ON DAMAge eVolution CURVES}

The methods described in the preceding sections allow calculation of the failure probability for known damage and fluence distributions. While they provide probabilities based on the onset of damage, they give no information on the severity of the local damage caused. Information on the severity of damage at onset and how it evolves on subsequent shots is of paramount importance in determining the functionality, or long term survivability of an optic on NIF. In the following sections the results of KDP damage testing on the Optical Sciences Laser are summarized and the method for determining the severity of damage to NIF triplers is described.

\subsection{KDP testing on the Optical Sciences Laser}

As described in reference [2], a laser conditioning and damage evolution study of $\mathrm{KDP}$ and $\mathrm{KD} * \mathrm{P}$ at $351 \mathrm{~nm}$ was recently undertaken on the Optical Sciences Laser (OSL). The primary goals of the campaign were to study stepwise KDP laser conditioning and damage evolution in a large beam environment. The test samples included material of 
rapidly grown KDP and KD*P boules 214 and DKDP11 respectively and conventionally grown, Beamlet vintage KD*P from boule LL6. The samples were exposed to $1 / 1,4 / 1,8 / 1$ and $12 / 1$ shot sequences to approximately equal final fluences. The damage sites were then scatter mapped to determine the local pinpoint density which was then compared to the local fluence in the beam. The data showed that damage evolution could be modeled by an exponential function of the form $N=N_{0} \exp (b F)$ where $N_{0}$ and $b$ are fitting parameters representing an initial defect concentration and slope respectively. The conditioning procedure yielded a maximum threshold increase of $1.4 \mathrm{X}$ which was achieved in the 8 shot ramps. The damage density continued to drop in $12 / 1$ exposures. It was also noted that the size distribution of damage pinpoints did not vary as a function of incident fluence, and once formed a damage pinpoint was stable against further, higher irradiation. This is shown in Figure 4.

The OSL test results were also correlated to the Zeus automated test results and showed good agreement in terms of threshold, degree of conditioning and relative performance among the samples. Table 2 shows the curve fit parameters for the samples tested. These pinpoint evolution curves will be used do calculate the expected damage behavior of KDP and KD*P when exposed to the NIF spatial profiles.

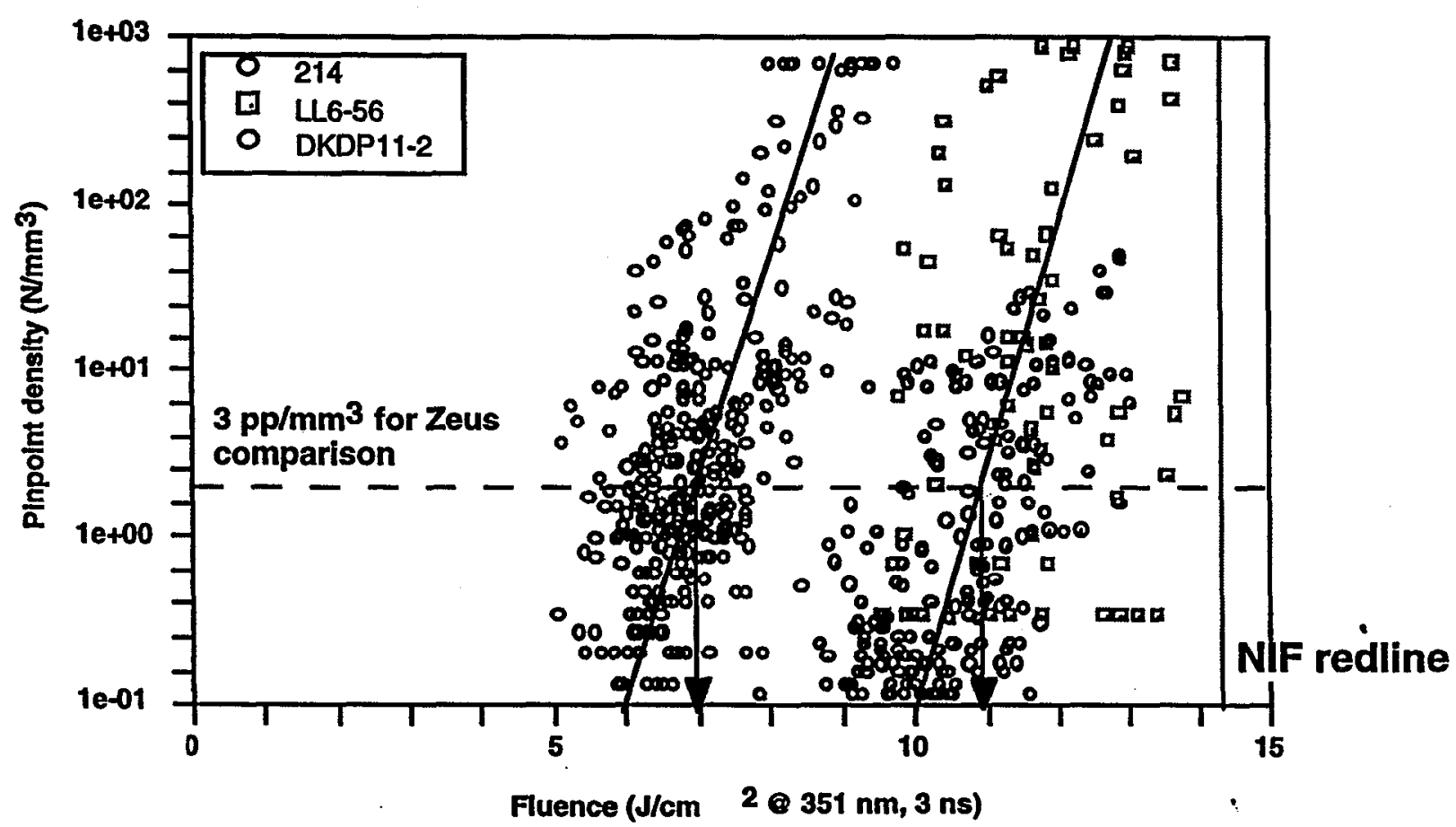

Figure 4. Danage evolution plot of 8/1 ramped exposure sequences for samples DKDP11, KDP 214 and LL6-56 tested on OSL. The data follow exponential evolution.

Table 2. Fit parameters for $1 / 1$ and $8 / 1$ shot sequences for samples 214, LL6-56 and DKDP11. Error bars (not given) on the fit parameters are large due to the spread in the data.

\begin{tabular}{|c|c|c|c|}
\hline Sample & Ramp sequence & $\mathbf{N}_{0}$ (pinpoints $/ \mathrm{mm}^{3}$ ) & b \\
\hline \hline \multirow{2}{*}{214} & $1 / 1$ & $3 \times 10^{-6}$ & 1.75 \\
\cline { 2 - 4 } & $7 / 1$ & $5 \times 10^{-10}$ & 2.0 \\
\hline \multirow{2}{*}{ LL6-56 } & $1 / 1$ & $1.5 \times 10^{-5}$ & 1.5 \\
\cline { 2 - 4 } & $8 / 1$ & $5 \times 10^{-10}$ & 2.0 \\
\hline \multirow{2}{*}{ DKDP11 } & $1 / 1$ & $7.5 \times 10^{-5}$ & 1.5 \\
\cline { 2 - 4 } & $8 / 1$ & $10^{-7}$ & 2.5 \\
\hline
\end{tabular}




\subsection{Damage evolution for NIF spatial profiles}

In order to calculate the damage expected on normal and redline NIF shots, the damage evolution curves must be applied to the model spatial profile on a cell-by-cell basis. Straightforward multiplication of the beam fluence in a cell with the damage evolution curve gives the local pinpoint density generated. Additional multiplication by the cell volume $\left(0.0095 \mathrm{~cm}^{3}\right)$ yields the number of pinpoints generated, which is intuitively easier to grasp. Figure 5 shows the difference in damage level for sample 214 (and LL6-56) generated by the NIF redline shot for unconditioned and well-conditioned material. The results of calculations for all the samples tested are given in Table 3 , which includes both standard and redline shots.

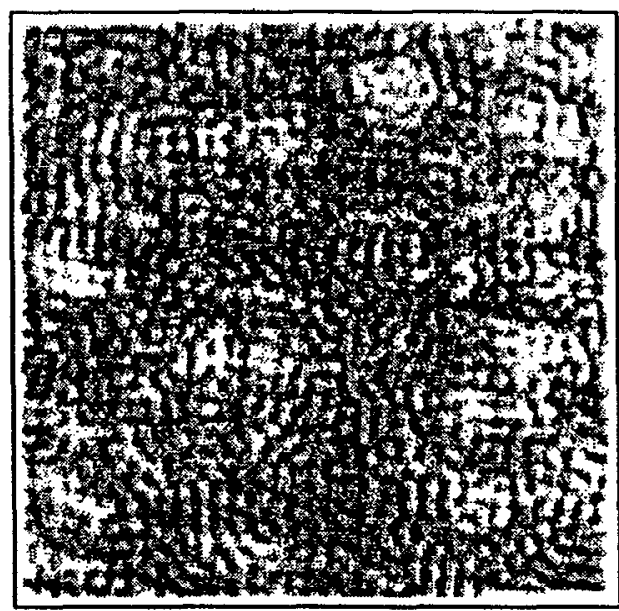

Sample 214 - unconditioned

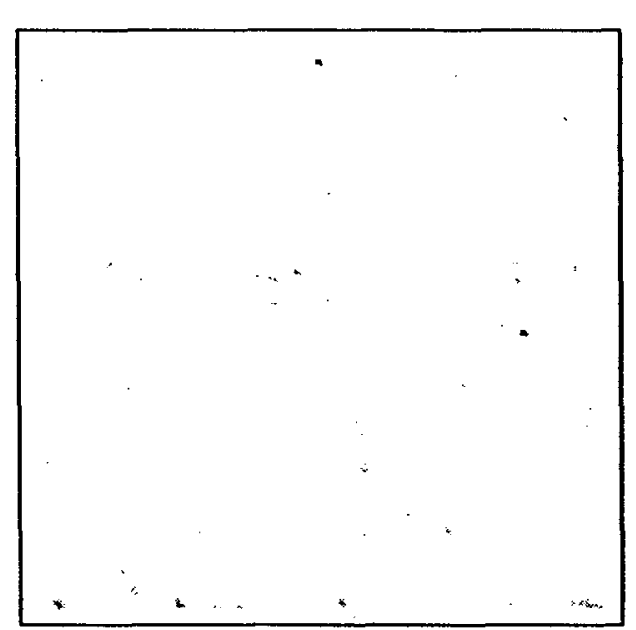

Conditioned

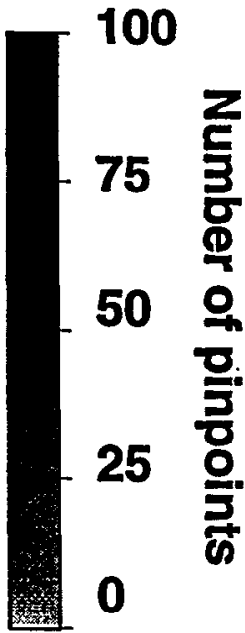

Figure 5. Plots representing the number of pinpoints formed in the bulk of KDP 214 or LL6-56 material for the NIF redline fluence spatial profile. The black areas represent densities which exceed 100 pinpoints in the 0.0095 $\mathrm{cm}^{3}$ cell volume. The color scale is the same for both images.

Table 3. Predicted maximum number of pinpoints generated by NIF spatial profiles for samples tested on QSL.

\begin{tabular}{|c|c|c|c|}
\hline Sample & $\begin{array}{c}\text { Conditioning } \\
\text { sequence }\end{array}$ & $\mathrm{N}_{\max }$ (std NIF) & $\mathrm{N}_{\max }$ (NIF redline) \\
\hline \hline \multirow{2}{*}{ DKDP11 } & $1 / 1$ & 2147 & 19858 \\
\cline { 2 - 4 } & $8 / 1$ & $1.09 \times 10^{6}$ & $4.1 \times 10^{7}$. \\
\hline \multirow{2}{*}{ KDP 214 } & $1 / 1$ & 2133 & 28086 \\
\cline { 2 - 4 } & $8 / 1$ & 9 & 165 \\
\hline \multirow{2}{*}{ LL6-56 } & $1 / 1$ & 429 & 3927 \\
\cline { 2 - 4 } & $8 / 1$ & 9 & 165 \\
\hline
\end{tabular}

Inspection of Table 3 shows that samples 214 and LL6-56 exhibit similar damage behaviors and show reduction of pinpoint numbers by $\sim 200 \mathrm{X}$ between the conditioned and unconditioned cases. For standard NIF shots the level of damage in well conditioned, high damage threshold material is not expected to affect laser operation. DKDP11 on the other hand shows no conditioning and the highest level of damage of the samples. It is doubtful that its damage behavior would be acceptable for NIF operation.

\subsection{Scatter map of Beamlet $37 \mathrm{~cm}$ tripler}

To assess the impact of laser damage on the operation of $\mathrm{KD} * \mathrm{P}$ triplers under NIF-like operational circumstances the $37 \mathrm{~cm} \mathrm{KD*P}$ tripler (LL1-12) from Beamlet was scatter mapped on the PLATO damage test system. This tripler had been "activated" in sequence of about 30 shots at a ramp rate of $\sim 0.3 \mathrm{~J} / \mathrm{cm}^{2} / \mathrm{shot}$ and is well conditioned based on the 
results of OSL testing given above. The mapping was undertaken to help resolve the apparent paradox between the apparent lack of visible bulk damage to the tripler and the high fluence shots produced with it. The bulk scatter diagnostic described in [1] was adapted to the PLATO system but the voltage response of the photomultiplier tube was not correlated to pinpoint density, as was the case for OSL experiments. This diagnostic was capable of detecting scatter sites on the order of a micron in diameter, which would otherwise be invisible to the naked eye at low concentrations. The results of the scatter map are shown in Figure 6.

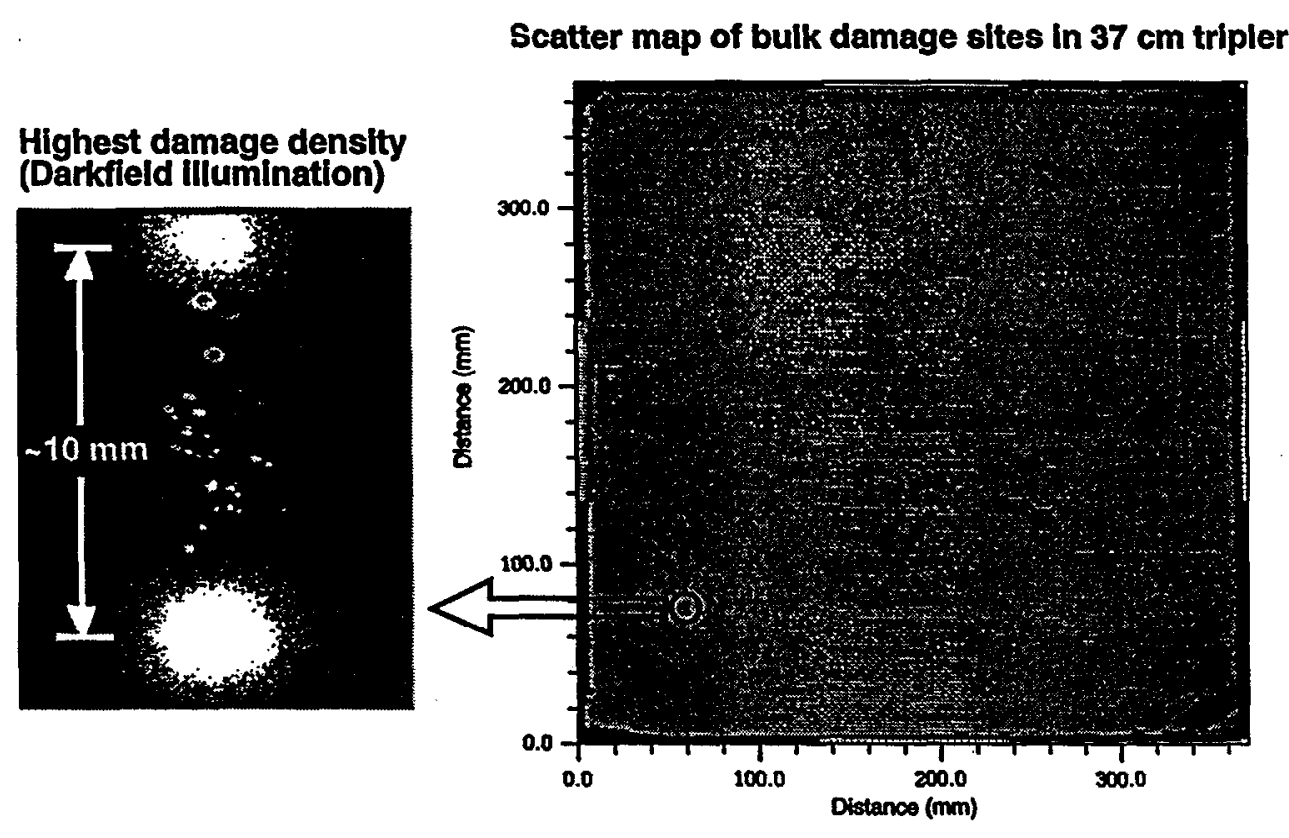

Figure 6. Bulk scatter map of Beamlet $37 \mathrm{~cm}$ tripler (LL1-12). The photograph shows the level of highest damage found in the tripler. It corresponds to a pinpoint density on the order of $0.5-1.0$ pinpoints $/ \mathrm{mm}^{3}$.

There are several features found in the scatter map. The highest density of pinpoints is found in the lower left comer. There are clear borders for the pinpoints along the left and bottom edges of the tripler which delineate the extent of the beam. A higher resolution image shows that the pinpoints form horizontal and vertical lines in this region and are associated with periodic spatial modulation of the beam fluence. The highest density of damage pinpoints occurs in this region, with a damage density of between 0.5 and 1 pinpoint per $\mathrm{mm}^{3}$. This density of pinpoints compares quite favorably with that predicted for LL6-56 material and the standard NIF shot (i.e. $\sim 1$ $\left.\mathrm{pp} / \mathrm{mm}^{3}\right)$. It is also noteworthy that the damage is not concentrated toward the output face of the crystal where $3 \omega$ fluence is the highest. This may have implications for the interpretation of single wavelength damage test results on doublers and triplers.

The next major concentration of pinpoints is in the upper central portion of the crystal. There is no clear boundary delineating the beam aperture here. It is likely that many of these pinpoints were the result of inclusions in the crystal from growth near the mush end. Based on the resolution of the scatter diagnostic, pinpoints formed by crystal growth or laser damage are indistinguishable in this area.

Because there is no information at hand regarding the actual spatial profile of the tripler's output on shots which may have caused damage, the response of the tripler to beam fluence cannot be measured. Since this tripler has similar damage response to LL6 material, it is possible to estimate that the peak $3 \mathrm{w}$ fluences which caused the maximum levels of damage are on the order of $13 \mathrm{~J} / \mathrm{cm}^{2}$. Since Beamlet operations have not been impacted by this level of tripler damage, it is safe to say that this material would also perform without incident on the NIF. 


\section{TOWARDS DETERMINING A FUNCTIONAL DAMAGE THRESHOLD FOR NIF KD*P}

The work described in this paper and references [1] and [2] represent the groundwork for specifying a functional damage threshold for KD*P triplers on the NIF. The results of Zeus automated tests, OSL conditioning studies and model NIF spatial profiles indicate that there is a low probability of damage occurring in high damage threshold $\mathrm{KD} * \mathrm{P}$. When damage does occur (in a well conditioned tripler), it will be minor, even for the case of a NIF redline shot. For unconditioned triplers the level of damage is much higher and it remains to determine an acceptable level. Several steps are required to do this. Since damage distribution and conditioning curves now exist, it should be possible to incorporate damage evolution more completely into modeling codes such as PROP92. To a first approximation the pinpoint can be modeled as an obscuration which scatters energy out of the beam, thereby reducing the conversion efficiency as this is probably the most important effect caused by bulk damage in the triplers. The impact on local beam propagation and transmitted wavefront also needs to be assessed. The levels of damage on the Beamlet tripler probably have no impact on the frequency conversion and downstream beam propagation, or formation of hotspots, however, the calculations are needed to provide quality assurance specifications on the triplers during production. These calculations are outside the scope of the KDP laser damage group and require a dedicated modeling effort.

In addition, the connection between the Zeus laser damage distribution and the damage evolution curves needs to be made for any sample tested in the future. This will involve determining the pinpoint density at each test site over the range of fluences applied during the ramp and generating a damage evolution curve akin to those for the OSL tests.

Furthermore, experiments are required to determine whether the damage response differs between the standard, single wavelength damage test and actual 3 color mixing which occurs in the tripler. This question arose because of the uniform distribution of damage observed in the Beamlet tripler. It was expected that damage would be observed primarily near the output face of the THG due to the high $3 \omega$ fluences developed there. Because the $1 \omega$ and $2 \omega$ thresholds are nominally $2 X$ higher than for $3 \omega$, is has long been thought that neither would contribute to damage in the tripler and therefore that single wavelength $3 \omega$ testing would be adequate to predict tripler damage response. Because of the statistical nature of laser damage it is quite possible that there is enough $1 \omega$ or $2 \omega$ fluence in parts of the beam to cause damage near the input face of the tripler. Investigation of this question involves either a dedicated campaign on OSL or a dedicated experimental campaign on Zeus at the very minimum.

\section{SUMMARY}

The results of Zeus automated tests, OSL conditioning studies and model NIF spatial profiles presented in this paper indicate that there is a low probability of damage occurring in high damage threshold $\mathrm{KD} * \mathrm{P}$ triplers. When damage occurs in a well conditioned tripler it will be minor, even for the case of a NIF redline shot and is not likely to affect operation of the laser. For unconditioned triplers the level of damage is much higher and it remains to determine an acceptable level.

The calculational methods presented here can be applied to other damage problems as well. The overlap of probability distributions gives the likelihood that damage will occur. The failure probabilities can be calculated if the damage distribution can be modeled by know functions, or in the case where there is a large amount of data in the overlap region, calculated numerically. On the other hand the failure probability gives no information on the severity of damage. For this, damage evolution models for specific optical components are needed. They can be applied to NIF model spatial profiles to determine the level of damage produced.

The authors would like to acknowledge Stephen Maricle and Mark Kozlowski for their assistance in scatter mapping the $37 \mathrm{~cm}$ Beamlet tripler on the PLATO system.

Work performed under the auspices of the U.S. Department of Energy by Lawrence Livermore National Laboratory under Contract No. W-7405-ENG-48. 


\section{REFERENCES}

1. M. Runkel, M. Yan, J. DeYoreo, N. Zaitseva "The effect of impurities and stress on the damage distributions of rapidly grown KDP crystals," in Laser Induced Damage in Optical Materials, SPIE Proceedings, Volume 3244, edited by G. Exarhos et. al, 211-222, SPIE, Bellingham, WA. 1997

2. M. Runkel, J. DeYoreo, W. Sell, D. Milam, "Laser conditioning study of KDP on the Optical Sciences Laser using large area beams," in Laser Induced Damage in Optical Materials. SPIE Proceedings, Volume 3244, edited by G. Exarhos et. al, 51-63, SPIE, Bellingham, WA. 1997

3. M. Runkel, "Monte Carlo simulation of the $R / 1$ automated damage test," in Laser Induced Damage in Optical Materials. SPIE Proceedings, edited by G. Exarhos et. al, SPIE, Bellingham, WA. 1998. A section of this paper describes the results of an $8 \times 100$ site test on KDP where the damage distribution is better modeled by a lognormal function.

4. ICF Quarterly Report 6(4), Special Issue, "Computational advances in ICF," Lawrence Livermore National Laboratory, Livermore, CA, UCRL-LR-105821-96-4, 1996

5. R. A. Sacks, M. A. Henesian, S. W. Haney, J. B. Trenholme, "The PROP92 Fourier beam propagation code," ICF Quarterly Report 6(4), Special Issue, "Computational advances in ICF," 207-213, Lawrence Livermore National Laboratory, Livermore, CA, UCRL-LR-105821-96-4, 1996

6. E.B. Haugen, Probabilistic Mechanical Design, John Wiley and Sons, New York, 1980

7. S. Zacks, Introduction to Reliability Analysis, Springer Verlag, New York, 1992

8. W.H. von Alven, Editor, Reliability Engineering, Prentice Hall, Inc., Englewood Cliffs, New Jersey, 1964

9. Care must be taken when evaluating (1) using tables. For example, to calculate equation (1) based on tables of $\operatorname{erf}(x)$ over $[-\infty, x]$ note that $P=1-\operatorname{erf}(x)$.

10. This derivation is equivalent to proving that the difference of two normally distributed functions is itself a normal function

\section{APPENDIX 1. DEVELOPMENT OF THE FAILURE PROBABILITY INTEGRAL}

The discussion that follows will be a description of how to calculate the survival probability associated with the overlap of beam fluence and damage distributions. There are two calculational approaches. The first considers the case where both the beam fluence and damage distributions are well modeled by gaussian functions, as is the case here. The second approach is a more general calculational prescription and can be applied to cases where the fitting function is non-gaussian, or numerical calculation is desired. Both methods draw heavily on the references cited, notably [1] and [2]. Details of the calculations not shown can be found there.

\section{A1.1 Gaussian probability density functions}

A gaussian probability density distribution is defined by

$$
f(x)=\frac{1}{\sqrt{2 \pi} \sigma} e^{-(x-\mu) / 2 \sigma^{2}}
$$

Herc, $\mu$ is the mean of the distribution and $\sigma$ is the standard deviation and gives a measure of the width of the distribution. It has the following properties: 
1) The probability of a measurement yielding an answer $X$ between $x$ and $x+d x$ is given by

$$
P(X)=f(X) d x
$$

2) The probability of a measurement yielding an answer $X$ in the interval $[a, b]$ is

$$
P(a<X<b)=\int_{a}^{b} f(x) d x
$$

3) The distribution is normalized such that its area is one. Note that the factor $1 / \sigma(2 \pi)^{1 / 2}$ scales the distribution so the normalization condition (4) holds, although the normalization factor is not used in fitting the actual damage and fluence distributions. As will be shown, the reliability integral does not depend on the normalization factor.

$$
\int_{-\infty}^{\infty} f(x) d x=1
$$

To calculate the probability of damage based on the gaussian fluence and damage distributions, the composite probability density function must be considered. It is

$$
d P_{\text {comp }}=f(B) F(D) d B d D
$$

Here, $B$ denotes the beam fluence distribution and $\mathrm{D}$ denotes the damage threshold distribution of the optic. To evaluate the probability that $B$ exceeds $D$ over the fluence range $[-\infty, \infty]$, it is necessary to transform variables so that one variable depends on the other. This is achieved by letting $Z=B-D$. The composite probability density can now be written as

$$
g(z) d z=\int_{-\infty}^{\infty} f(B) F(B-z) d B d z
$$

By equations (2) and (3), the probability that the beam fluence will exceed the damage threshold is given by

$$
P(B>D)=\int_{-\infty}^{\infty} g(z) d z
$$

Using gaussian expressions (1) for B and D in (6) yields a more explicit form for $\mathrm{g}(\mathrm{z})$ :

$$
g(z)=\frac{1}{2 \pi \sigma_{B} \sigma_{D}} \int_{-\infty}^{\infty} \exp \left[-\frac{\left(B-\mu_{B}\right)^{2}}{2 \sigma_{B}{ }^{2}}-\frac{\left(B-Z-\mu_{D}\right)^{2}}{2 \sigma_{D}{ }^{2}}\right] d B .
$$

Following [3], $g(z)$ can be simplified to an explicit function of $z$ only. This requires substantial algebraic manipulation and does not add to the discussion so will be skipped ${ }^{4}$. The result is: 


$$
g(z)=\frac{1}{\sqrt{2 \pi\left({\sigma_{B}}^{2}+{\sigma_{D}}^{2}\right)}} \exp \left[-\frac{\left(z+\mu_{D}-\mu_{B}\right)^{2}}{2\left({\sigma_{B}}^{2}+{\sigma_{D}}^{2}\right)}\right]
$$

The probability that fluence in the beam exceeds the damage threshold (7) is now given by

$$
P(B>D)=\int_{z=B-D \geq 0}^{\infty} g(z) d z
$$

Note that $\mu_{\mathrm{Z}}=\mu_{\mathrm{B}}-\mu_{\mathrm{D}}$ and $\sigma_{\mathrm{z}}^{2}=\sigma_{\mathrm{B}}{ }^{2}+\sigma_{\mathrm{D}}{ }^{2}$. The variable transformation

$$
t^{2}=\frac{\left(z+\mu_{D}-\mu_{B}\right)^{2}}{\left(\sigma_{B}^{2}+\sigma_{D}^{2}\right)}
$$

allows the integral to be rewritten in a form from which it can be evaluated from standard tables, or calculated numerically*:

$$
P(B>D)=\frac{1}{\sqrt{2 \pi}} \int_{\frac{\mu_{z}}{\sigma_{z}}}^{\infty} e^{-t^{2} / 2} d t
$$

\section{A1.2 General calculational method}

The method described below can be applied to situations where the damage distribution is not gaussian. It lends itself to situations where the distribution can be modeled by a different functional form, or where direct numerical calculation of the data is desired. Figure 4 shows the overlap of arbitrary damage and beam fluence distributions.

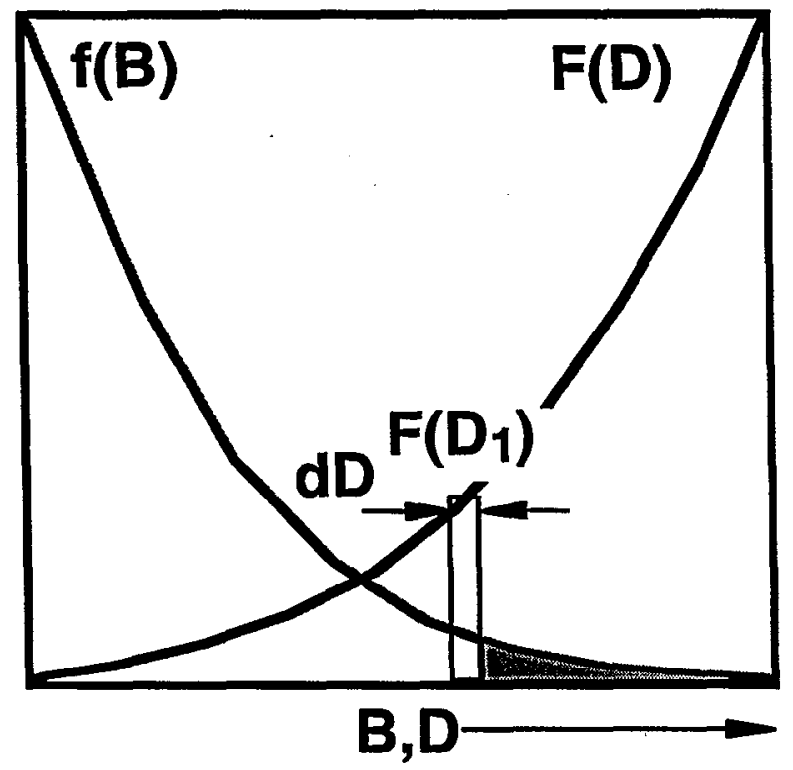

Figure 4. Overlap of beam fluence and damage distributions. 
Considering Figure 4, the probability that the beam fluence is greater than the damage threshold $(B>D)$ for any arbitrary threshold fluence $D_{1}$ is given by

$$
\delta P\left(D_{1}\right)=F\left(D_{1}\right) d D
$$

Damage will occur if the beam fluence equals or exceeds the (local) threshold i.e. $B \geq D$. This probability is shown as the shaded area under the $f(B)$ curve in Figure 4 and is given by:

$$
d P\left(B>D_{1}\right)=\int_{D_{1}}^{\infty} f(B) d B
$$

So, the total failure probability differential is given by the product of (13) and (14). Integrating over the range of D gives the total probability of failure for the two distributions:

$$
P_{f}(B>D)=\int_{-\infty}^{\infty} F(D) \int_{D}^{\infty} f(B) d B d D
$$

For evaluation of (15) it is necessary to substitute explicit functional forms for $F(D)$ and $f(B)$ and perform the integral over $f(B)$ first in order to obtain an explicit function of $D$ in the final integrand. This prescription can also be used to evaluate the data numerically, although accurate evaluation of test data in this manner would require a large number of data points at the toe of the damage distribution curve. Data of this resolution is not always available. This method of evaluating the failure probability has not been used for KDP. 